\title{
LOS USOS NO LeÑOSOS DE LOS ENCINOS EN MÉXICO
}

\author{
Azucena De Lourdes Luna-Josép , Linda Montalvo-Espinosa ${ }^{1}$ y Beatriz Rendón-Aguilar ${ }^{2}$ \\ ${ }^{1}$ Colegio de Posgraduados, Montecillos, México \\ ${ }^{2}$ Departamento de Biología, Universidad Autónoma Metropolitana-Iztapalapa. A.P. 55-535. \\ México, D.F. C.P. 09340. Fax 58-04-46-88. Correo-e: betyrendon@aol.com
}

\begin{abstract}
Resumen: Se presenta una revisión bibliográfica y de herbario de los usos no leñosos de algunos encinos en México. Cincuenta y cinco especies de Quercus se utilizan y obtienen exclusivamente mediante la recolección. La mayoría se emplea en los estados ubicados en el centro y sur del país. No se encontró una relación entre el número de especies utilizadas y la diversidad de especies por estado. Se registraron cinco categorías de uso: (1) medicinal, que se relaciona principalmente con problemas del aparato digestivo; (2) alimenticio, que comprende la elaboración y consumo de alimento fresco o procesado; (3) artesanal, para la elaboración de diferentes artículos como rosarios o juguetes; (4) forraje, principalmente para la alimentación de ganado porcino y caprino; (5) taninos y colorantes, para curtir piel, como mordiente y para teñir hilo. Existe una relación entre la presencia de grupos indígenas y el uso de los encinos, lo que indica su valor cultural, además de su importancia como recurso vegetal complementario.
\end{abstract}

Palabras clave: Quercus, encino, usos no leñosos, medicinal, alimenticio, forraje, artesanías, taninos, colorante, grupos étnicos.

\begin{abstract}
A review based on literature and herbarium voucher information on the non-wood uses of oaks in Mexico is presented. Fifty-five species of Quercus are gathered and used for different purposes, mainly in those states located in the central and south regions of the country. No relationship was found between species diversity by state and number of useful species. Five different uses were recorded: (1) medicinal, related mainly to digestive apparatus sicknesses: (2) food, including consumption of different structures, both fresh and processed; (3) handcraft, to elaborate different manufactured crafts, like rosaries and toys; (4) fodder, for goats and pigs; (5) tannins and dyes, to turning raw skins into leather and to act like mordant and to dye fibers. There is a relationship between the presence of ethnic groups and the use of the oaks in a given region, indicating the cultural value of these species in addition of their importance as a complementary plant resource.
\end{abstract}

Key words: Quercus, oak, non-wood uses, medicinal, food, fodder, handcraft, tanines, dyes, ethnic groups.

$\mathbf{L}$ os productos forestales no leñosos representan valores económicos, sociales y culturales importantes y se usan con fines de alimentación, medicinal y artesanal, entre otros. De acuerdo con la FAO (1999), los productos forestales no leñosos se refieren a los "bienes de origen biológico diferentes de la madera, derivados de bosques, terrenos arbolados y árboles situados fuera de los bosques" y comprenden específicamente a productos provenientes de raíces, tallos, corteza, hojas, agallas, flores y frutos. Dicha definición excluye, en consecuencia, el uso de madera, astillas, carbón y leña, así como herramientas, utensilios pequeños y figurillas elaborados de madera.

En México existe una gran variedad de productos no leñosos provenientes de diferentes especies, los cuales representan una fuente importante de materiales para diversos grupos étnicos y mestizos, tanto con fines de subsistencia como para comercialización (Adger et al., 1998). Entre estas especies destacan varios miembros del género Quercus (Fagaceae).

El género Quercus presenta su centro de diversificación en México, ya que de las 450 especies estimadas a nivel mundial, entre 135 y 150 se encuentran en nuestro país, y de ellas 86 se consideran endémicas (Nixon, 1998; Zavala, 1998). Los encinos representan uno de los componentes florísticos notables en diversas comunidades templadas y tropicales del país. Como arbustos forman parte de diversos matorrales y como árboles conforman comunidades vegetales características de las zonas montañosas de México.

Los estudios que han abordado el uso de los encinos en México resaltan su uso maderable debido a sus propiedades físicas, mecánicas y anatómicas. Estos atributos hacen de la madera un recurso adecuado para la elaboración de recipientes 
culinarios, pisos, postes, durmientes, chapa, embarcaciones, muebles, mangos para herramientas y partes de instrumentos musicales, entre otros (Reyes, 1995; de la Paz Pérez, 2000). A nivel nacional, la madera de encino ocupa el segundo lugar de aprovechamiento y los usos a los que se destina principalmente son: celulosa (54\%), escuadría (30\%), leña (7\%) y carbón (6\%) (de la Paz Pérez et al., 2000).

Si bien el uso maderable y los derivados de los encinos son ampliamente reconocidos, por el contrario su utilidad no maderable o no leñosa ha sido poco valorada, no obstante que en diversas comunidades étnicas y mestizas del país la obtención y elaboración de diversos productos medicinales o alimenticios forman parte de su cultura. Este hecho se constata al encontrar registro del uso de los encinos con fines medicinales en el Códice de la Cruz-Badiano (de la Cruz y Badiano, 1991).

En los trabajos que han sintetizado el uso de los encinos en México se menciona de manera general a los usos no maderables, sin profundizar en su descripción y en la identidad de las especies utilizadas (Zavala, 1990; González, 1993; Martínez, 1994; de la Paz Pérez, 2000). Hasta el momento el único registro antiguo encontrado que hace referencia a los usos no maderables es el de Ponce (1946). Sin embargo, dicha descripción la realiza con ocho especies de encinos que se distribuyen en diferentes partes del mundo; para México únicamente menciona y describe a $Q$. mellifera, que en los listados actuales no está registrada.

El objetivo del presente trabajo fue analizar los usos no leñosos de los encinos en México a partir de la revisión de literatura y de ejemplares de herbario. El análisis de la información se basó en: (1) la representatividad de especies de encino útiles y su relación con el número total especies de encino por estado, (2) los diversos usos, profundizando en aspectos como la parte útil de la planta, y (3) su utilidad entre diferentes grupos étnicos.

\section{Materiales y métodos}

La realización de este trabajo abarcó tres etapas. La primera consistió en la revisión de fuentes bibliográficas. Se revisaron trabajos etnobotánicos, taxonómicos y florísticos que especificaran algún uso no leñoso que se le dé al género Quercus, incluyendo las categorías medicinal, alimenticio, artesanal, forraje, taninos y colorantes. Para sistematizar esta información se consideró el estado de la República, el grupo étnico (si es que se menciona) y el uso. La información se registró textualmente, con excepción del nombre científico, el cual se actualizó con base en los trabajos de Valencia (1995) y Govaerts y Frodin (1998).

Durante la segunda etapa se realizó una revisión de los ejemplares botánicos del género Quercus depositados en tres herbarios: Herbario Metropolitano "Ramón Riba y Nava Esparza" de la Universidad Autónoma MetropolitanaIztapalapa (UAMIz); Herbario de la Escuela Nacional de
Ciencias Biológicas del Instituto Politécnico Nacional (ENCB) y el Herbario Nacional del Instituto de Biología de la Universidad Nacional Autónoma de México (MEXU). Se elaboró una base de datos que contiene el nombre de la especie, el lugar de colecta, el estado, el grupo étnico (si lo menciona), el colector y el número de colecta. Nuevamente, la información se registró tal y como estaba escrita en las etiquetas y únicamente se actualizaron los nombres científicos siguiendo las clasificaciones de Valencia (1995) y Govaerts y Frodin (1998).

La tercera etapa correspondió al análisis de datos, que consistió en comparar el número de: total de especies por estado/especies útiles por estado; especies utilizadas dentro de cada categoría de uso registrada; especies que tienen uno, dos o más usos (se registraron por especie hasta cinco usos); especies que se utilizan para curar diversos tipos de enfermedades; especies que se utilizan en alguna de las categorías y la parte o estructura de la planta más comúnmente utilizada con ese fin. Para analizar esta relación, se aplicó una prueba de $\chi^{2}$ para probar la hipótesis de que no hay una relación entre el uso y la estructura utilizada para dicho fin. Para el análisis se consideraron dos usos (medicinal, alimenticio), por ser los que concentran el mayor número de especies y cuatro estructuras (corteza, bellotas, hojas y otras). Finalmente, para obtener la proporción de especies utilizadas por cada grupo étnico se tomaron como base los registros derivados de la literatura y los herbarios sobre la presencia de determinado grupo étnico a nivel estatal, y se cuantificó el total de especies utilizadas asociadas a dicho grupo, que se definió como el número actual de especies útiles. La distribución se relacionó con el número de especies reportadas como utilizadas para dichos estados y se consideró como el número potencialmente utilizable. Se obtuvo una relación entre el número de especies actualmente útiles sobre el número potencialmente utilizable.

\section{Resultados}

Especies de encinos con usos no leñosos. Se registraron 55 especies útiles de encinos que incluyen nueve ejemplares no determinados, obtenidos de la literatura, nombrados como Quercus sp. Esto representa entre 36 y $40 \%$ de las especies consideradas para México (Nixon, 1998; Zavala, 1990, 1998). Cabe aclarar que en la información recopilada se mencionan algunas especies de Quercus cuya distribución no corresponde a la indicada por González (1993), así como los nombres científicos actualizados (cuadro 1) (Valencia, 1995; Govaerts y Frodin, 1998). De la literatura consultada se obtuvo un total de 44 especies. La revisión de herbarios arrojó un total de 26 especies que reportan algún uso (ENCB, 4 especies; MEXU, 25 especies y UAMIz, ninguno) (cuadro 1), de las cuales 11 fueron de registro exclusivo, es decir, no se mencionaron en la literatura revisada. De las 55 especies registradas, 19 (34.5\%) son endémicas para México (González, 1993) (cuadro 1). 
Cuadro 1. Especies de encino que presentan uso no maderable en México. Se reporta su distribución, grupos étnicos que las utilizan (cuando se indica), nombre común, uso(s) y fuentes de referencia.

\begin{tabular}{|c|c|c|c|c|c|}
\hline Especie $^{a}$ & Estado & Grupo étnicob $^{b}$ & Nombre común & Uso $^{\mathrm{c}}$ & $\begin{array}{l}\text { Fuentes de } \\
\text { referencia }^{d}\end{array}$ \\
\hline Q. acatenangensis Trel. & Chiapas & & & $M$ & Aguilar et al. (1994d) \\
\hline Q. acutifolia Née * & Guerrero, Chiapas & $\mathrm{N}$ & & M & Pérez (1999)', Negrete (1991) \\
\hline Q. affinis Schiede* & $\begin{array}{l}\text { Puebla, } \\
\text { Estado de México }\end{array}$ & $N, M$ & & $\mathrm{M}, \mathrm{A}, \mathrm{F}, \mathrm{Ar}$ & $\begin{array}{l}\text { Chino y Jacquez (1986), } \\
\text { Estrada (1996) }\end{array}$ \\
\hline Q. aff. eduardii Trel. & Estado de México & & encino & M & Linares $(1996)^{1}$ \\
\hline $\begin{array}{l}\text { Q. aff. emory Humb. } \\
\text { et Bonpl. }\end{array}$ & Estado de México & & $\begin{array}{l}\text { encino de hoja } \\
\text { delgada }\end{array}$ & M & Linares $(1994)^{1}$ \\
\hline $\begin{array}{l}\text { Q. aff. obtusata Humb. } \\
\text { et Bonpl. }\end{array}$ & Estado de México & & encino & M & Linares $(1994)^{1}$ \\
\hline Q. aff. persiifolia Liebm. & Veracruz & & & M & Vovides $(1976)^{1}$ \\
\hline Q. albocincta Trel. * & Chihuahua & $\mathrm{TR}, \mathrm{Te}$ & & A & Jiménez (1990) \\
\hline Q. arizonica Sarg. & Chihuahua & $\mathrm{Te}$ & & A & Jiménez (1990) \\
\hline Q. brandegeei Goldman * & & & & A & Jiménez (1990) \\
\hline Q. candicans Née & $\begin{array}{l}\text { Oaxaca, Chiapas, } \\
\text { Michoacán, Veracruz }\end{array}$ & Z & & $\mathrm{M}, \mathrm{A}, \mathrm{F}, \mathrm{Ar}, \mathrm{TC}$ & $\begin{array}{l}\text { Santíz (1987)', Tovar }(1988)^{1} \text {, } \\
\text { Bello y Labat (1987), Jiménez } \\
\text { (1990), Luna (2001) }\end{array}$ \\
\hline Q. castanea Née & $\begin{array}{l}\text { Puebla, Morelos, } \\
\text { Guerrero, Michoacán }\end{array}$ & $\mathrm{N}$ & $\begin{array}{l}\text { tepezcohuite, } \\
\text { encino negro }\end{array}$ & $\mathrm{M}, \mathrm{F}, \mathrm{Ar}$ & $\begin{array}{l}\text { Viveros y Casas (1983)1, Cruz } \\
\text { y García (1995)', Bello y Labat } \\
\text { (1987), Castro (1988), Jiménez } \\
\text { (1990), Maldonado (1997) }\end{array}$ \\
\hline Q. conspersa Benth. & $\begin{array}{l}\text { Puebla, Oaxaca, } \\
\text { Michoacán }\end{array}$ & $\mathrm{N}, \mathrm{Z}$ & & $\mathrm{M}, \mathrm{TC}$ & $\begin{array}{l}\text { Bello y Labat (1987), } \\
\text { Aguilar et al. (1994a) }\end{array}$ \\
\hline $\begin{array}{l}\text { Q. corrugata Hook. [como } \\
\text { Q. cylobalanoides] }\end{array}$ & & & & $\mathrm{M}, \mathrm{A}$ & Jiménez (1990) \\
\hline $\begin{array}{l}\text { Q. crassifolia Humb. } \\
\text { et Bonpl. }\end{array}$ & $\begin{array}{l}\text { Chihuahua, Estado de } \\
\text { México, Morelos, } \\
\text { Chiapas, Veracruz, } \\
\text { Michoacán }\end{array}$ & $\mathrm{N}, \mathrm{TR}, \mathrm{O}, \mathrm{Te}, \mathrm{M}$ & & $\mathrm{M}, \mathrm{A}, \mathrm{TC}$ & $\begin{array}{l}\text { Luna (1989), Aguilar et al. } \\
\text { (1994a), Aguilar et al. } \\
\text { (1994b), Berenice (2001) }\end{array}$ \\
\hline $\begin{array}{l}\text { Q. crassipes Humb. } \\
\text { et Bonpl. * }\end{array}$ & $\begin{array}{l}\text { Tlaxcala, Estado de } \\
\text { México, Michoacán }\end{array}$ & $\mathrm{N}, \mathrm{O}, \mathrm{Pu}$ & & $\mathrm{M}, \mathrm{A}, \mathrm{F}, \mathrm{Ar}, \mathrm{TC}$ & $\begin{array}{l}\text { Bello y Labat (1987), } \\
\text { Jiménez (1990), Aguilar et al. } \\
\text { (1994c), Sánchez (1996), } \\
\text { Espejel et al. (1999) }\end{array}$ \\
\hline Q. chihuahuensis Trel. * & Chihuahua & $\mathrm{Te}$ & & A & Jiménez (1990) \\
\hline Q. deserticola Trel. & Jalisco & & encino chino & $\mathrm{M}, \mathrm{A}, \mathrm{TC}$ & $\begin{array}{l}\text { V. de Puga }(1966)^{2} \text {, Zavala et } \\
\text { al. (1999) }\end{array}$ \\
\hline Q. devia Goldman* & & & & A & Jiménez (1990) \\
\hline Q. durifolia Seem * & Chihuahua & $\mathrm{TR}, \mathrm{Te}$ & & A & Jiménez (1990) \\
\hline Q. elliptica Née & $\begin{array}{l}\text { Jalisco, Veracruz, } \\
\text { Michoacán }\end{array}$ & To, $\mathrm{T}$ & $\begin{array}{l}\text { encino rojo (cucat, } \\
\text { totonaco) }\end{array}$ & $\mathrm{M}, \mathrm{Ar}$ & $\begin{array}{l}\text { Bello y Labat (1987), } \\
\text { Evangelista(1980)' }{ }^{1} \text { González } \\
(1983)^{2} \text {, Morales y Toledo } \\
\text { (1987), López (1988) }\end{array}$ \\
\hline Q. emoryi Torr. & Chihuahua & $\mathrm{T}, \mathrm{R}$ & & A & Jiménez (1990) \\
\hline Q. glabrescens Benth. * & $\begin{array}{l}\text { Morelos, Puebla, } \\
\text { Guerrero, Estado de } \\
\text { México }\end{array}$ & M & & $M, F$ & $\begin{array}{l}\text { Estrada (1996), Zavala et } \\
\text { al. (1999) }\end{array}$ \\
\hline $\begin{array}{l}\text { Q. glaucescens Humb. } \\
\text { et Bonpl. }\end{array}$ & Veracruz, Oaxaca & $\mathrm{P}$ & $\begin{array}{l}\text { encino amarillo } \\
\text { (puutx soj, popoluca) } \\
\text { encino blanco (poop } \\
\text { xoj, poop xoj aky) }\end{array}$ & M & $\begin{array}{l}\text { Antonio y Wiss }(1986,1988)^{1} \\
\text { Leonti }(2000)^{1}\end{array}$ \\
\hline $\begin{array}{l}\text { Q. glaucescens Trel. } \\
{[\text { como Q. sintetica*] }}\end{array}$ & Oaxaca & Mi & & M & Aguilar et al. (1994a) \\
\hline Q. glaucoides Mart. et Gal. & $\begin{array}{l}\text { Guerrero, Morelos, } \\
\text { Michoacán }\end{array}$ & $\mathrm{N}, \mathrm{Mt}, \mathrm{Pu}$ & $\begin{array}{l}\text { encino prieto, } \\
\text { encino chaparro }\end{array}$ & $M, A, F$ & $\begin{array}{l}\text { Viveros y Casas }(1983 a)^{1}{ }^{1} \\
\text { Viveros y Casas }(1983 b)^{1}, \\
\text { Viveros y Casas (1985), } \\
\text { Jiménez (1990), Maldonado } \\
\text { (1997), Gómez (2000) }\end{array}$ \\
\hline Q. grahamii Benth. & Guerrero, Oaxaca & & $\begin{array}{l}\text { encino tepezcohuite, } \\
\text { encino de tinta }\end{array}$ & M & $\begin{array}{l}\text { Ramírez (s/f)' }{ }^{1} \text { Calzada } \\
(1994)^{1}\end{array}$ \\
\hline Q. greggii Trel. * & Hidalgo & & & M & \\
\hline Q. hypoleucoides A.Camus & Chihuahua & $\mathrm{TR}, \mathrm{Te}$ & & A & Jiménez (1990) \\
\hline Q. ilex $\mathrm{L}$. & Morelos & & & M & García (1992) \\
\hline Q. insignis Mart. et Gal. & Jalisco & & & $\mathrm{F}$ & González (1986) \\
\hline
\end{tabular}


Cuadro 1. Continuación

\begin{tabular}{|c|c|c|c|c|c|}
\hline Especie $^{a}$ & Estado & Grupo étnico $^{b}$ & Nombre común & Uso $^{c}$ & $\begin{array}{l}\text { Fuentes de } \\
\text { referencia }^{d}\end{array}$ \\
\hline Q. laeta Liebm. * & Estado de México & & & M & Aguilar et al. (1994a) \\
\hline Q. lancifolia Schltdl. et Cham. & Veracruz & & & M & Vovides $(1976)^{1}$ \\
\hline Q. laurina Humb. et Bonpl. * & $\begin{array}{l}\text { Estado de México, } \\
\text { Michoacán }\end{array}$ & $\mathrm{O}, \mathrm{M}$ & $\begin{array}{l}\text { encino de hoja } \\
\text { delgada }\end{array}$ & $\mathrm{M}, \mathrm{A}, \mathrm{F}, \mathrm{Ar}$ & $\begin{array}{l}\text { Linares }(1994 a)^{1} \text {, Linares } \\
(1994 b)^{1} \text {, Aguilar et al. } \\
(1994 a), \text { Berenice }(2001), \\
\text { Bello y Labat }(1987)\end{array}$ \\
\hline Q. magnoliifolia Née & $\begin{array}{l}\text { Estado de México, } \\
\text { Nayarit, Michoacán, } \\
\text { Oaxaca }\end{array}$ & $\mathrm{Pu}$ & & $M, F$ & $\begin{array}{l}\text { Matuda (1952)', Soto (1984)', } \\
\text { Santos (2000)', Aguilar et al. } \\
\text { (1994c), Gómez (2000) }\end{array}$ \\
\hline $\begin{array}{l}\text { Q. mexicana Humb. } \\
\text { et Bonpl. * }\end{array}$ & Hidalgo, Puebla & & encino & $\mathrm{M}, \mathrm{Ar}$ & $\begin{array}{l}\text { Tlapa y Ubierna }(1987)^{1} \text {, } \\
\text { Barquín }(1989)^{1}\end{array}$ \\
\hline Q: microphylla Née * & Durango, Hidalgo & & encino blanco & $\mathrm{M}, \mathrm{F}, \mathrm{Ar}$ & Martínez y Saldivar (1976) \\
\hline Q. muehlenbergii Engelm. & Morelos & $\mathrm{N}$ & & M & Gómez y Chong (1985) \\
\hline Q. oblongifolia Torr. & & & & A & Jiménez (1990) \\
\hline $\begin{array}{l}\text { Q. obtusata Humb. } \\
\text { et Bonpl. * }\end{array}$ & $\begin{array}{l}\text { Guerrero, Tlaxcala, } \\
\text { Estado de México, } \\
\text { Michoacán, Nayarit }\end{array}$ & Mt & $\begin{array}{l}\text { encino, roble (tuá), } \\
\text { encino chimeco } \\
\text { (thiahta, chimeco) }\end{array}$ & $M, A, F$ & $\begin{array}{l}\text { Viveros y Casas (1983)' } \\
\text { Vega }(1990)^{1}, \text { Linares } \\
(1994)^{1}, \text { Viveros y Casas } \\
(1985), \text { Bello y Labat (1987), } \\
\text { Jiménez (1990), Espejel et al. } \\
\text { (1999) }\end{array}$ \\
\hline Q. oleoides Schltdl. et Cham. & $\begin{array}{l}\text { Veracruz, Oaxaca, } \\
\text { Hidalgo, Puebla }\end{array}$ & To, T, Mi & $\begin{array}{l}\text { encino blanco (pop } \\
\text { xoj, popoluca, } \\
\text { encino, encino prieto } \\
\text { (jing xoj), pop xoj }\end{array}$ & M & $\begin{array}{l}\text { Calzada y Castillo }(1976)^{1}, \\
\text { Antonio y Wiss }(1986)^{1}, \\
\text { Heinrich (1986)', Martínez } \\
(1988)^{1} \text {, Frei }(1992)^{1}, \text { Leonti } \\
(1999)^{1}, \text { Zamora y Hernández } \\
\text { (1985), López (1988), Antonio } \\
\text { (1989), Martínez (1992), } \\
\text { Aguilar et al. (1994d) }\end{array}$ \\
\hline Q. peduncularis Née & $\begin{array}{l}\text { Chiapas, Guerrero } \\
\text { Oaxaca, Veracruz, }\end{array}$ & $\mathrm{N}, \mathrm{Mi}, \mathrm{P}$ & $\begin{array}{l}\text { encino negro (yyk } \\
\text { soj, popoluca) }\end{array}$ & $M, A$ & $\begin{array}{l}\text { Gómez }(1988)^{1}, \text { Leonti } \\
(2000)^{1}, \text { Negrete (1991), } \\
\text { Aguilar et al. (1994a) }\end{array}$ \\
\hline Q, perseafolia Liebm. & Oaxaca & & $\begin{array}{l}\text { encino colorado } \\
\text { (tsap xoj) }\end{array}$ & M & Antonio y Wiss (1986) ${ }^{1}$ \\
\hline Q. resinosa Liebm. & Michoacán & $\mathrm{Pu}$ & & M & Gómez (2000) \\
\hline Q. rugosa Née & $\begin{array}{l}\text { Chihuahua, Distrito } \\
\text { Federal, Estado de } \\
\text { México, Puebla, } \\
\text { Morelos, Michoacán }\end{array}$ & $\mathrm{N}, \mathrm{O}, \mathrm{Te}, \mathrm{M}$ & encino rojo & $M, A, F$ & $\begin{array}{l}\text { Bye y Linares }(1998)^{1} \text {, } \\
\text { Chino y Jacquez (1986), } \\
\text { Bello y Labat (1987), Jiménez } \\
\text { (1990), Estrada (1996), Zavala } \\
\text { et al. (1999), Berenice (2001) }\end{array}$ \\
\hline Q. sapotiifolia Liebm. & Chiapas, Oaxaca & & $\begin{array}{l}\text { tz'otz'op tulan } \\
\text { encino blanco } \\
\text { (poop xoj) }\end{array}$ & M & $\begin{array}{l}\text { Antonio y Wiss }(1986)^{1} \text {, } \\
\text { Hernández }(1988)^{1}\end{array}$ \\
\hline Q. sartorii Liebm. & Guerrero & & tepezcohuite & M & Cruz y García (1994)¹ \\
\hline $\begin{array}{l}\text { Q. scytophylla Liebm. } \\
\text { [como Q. epileuca]* }\end{array}$ & & & & A & Jiménez (1990) \\
\hline Q. sempervirens & Estado de México & & & M & Salgado (1978) \\
\hline $\begin{array}{l}\text { Q. sideroxyla Humb. et } \\
\text { Bonpl. [como Q. omissa] }\end{array}$ & Chihuahua & $\mathrm{Te}$ & & A & Jiménez, (1990) \\
\hline $\begin{array}{l}\text { Q. tuberculata Liebm. } \\
\text { [como Q. idonea] }\end{array}$ & & & & A & Jiménez (1990) \\
\hline Q. urbanii Trel. * & Guerrero & Mt & encino & $A, F$ & $\begin{array}{l}\text { Viveros y Casas ( 1985), } \\
\text { Jiménez (1990) }\end{array}$ \\
\hline Q. urbanii aff. parvifolia Trel. & Oaxaca & & & $\mathrm{M}, \mathrm{TC}$ & MacDougall (1952) \\
\hline Q. xalapensis Bonpl. & Veracruz & $P$ & ok soj & M & Leonti $(2000)^{1}$ \\
\hline Quercus sp. & $\begin{array}{l}\text { Michoacán, Hidalgo, } \\
\text { Morelos, Oaxaca, } \\
\text { Nuevo León, Sonora, } \\
\text { Puebla, Guerrero }\end{array}$ & N, Mi, Z & & $\mathrm{M}, \mathrm{F}, \mathrm{Ar}$ & $\begin{array}{l}\text { Sandoval (1977), García (1981), } \\
\text { Antonio (1989), Luna (1989), } \\
\text { Aguilar et al. (1994a), Avilés } \\
\text { (1985), Aguilar et al. (1994b, c) }\end{array}$ \\
\hline
\end{tabular}

a * Especies endémicas para México

b $\quad($ Mazahua $=$ M, Mixe $=$ Mi, Mixteco $=$ Mt, Náhuatl= N, Otomí $=O$, Popoluca $=P$, Purépecha $=P u$, Tarahumara o Rarámuri $=T R$, Tepehua $=T$, Tepehuán $=\mathrm{Te}$, Totonaco $=\mathrm{To}, \mathrm{Zapoteco}=\mathrm{Z}$

c $M=$ Medicinal, $A=$ Alimenticio, $F=$ Forraje, $A r=$ Artesanal, $T C=$ Taninos y colorantes

d $1=$ MEXU, 2= ENCB; las citas sin número corresponden a fuentes bibliográficas 


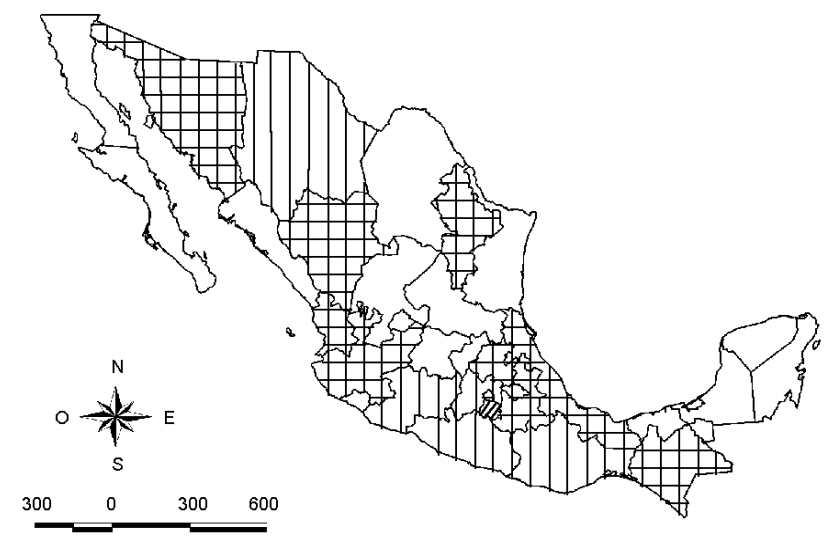

Figura 1. Estados de la República Mexicana en donde se reporta el uso no leñoso de al menos una especie del género Quercus. Líneas verticales, 1 - 20\% de especies útiles; cuadrícula, 20.1\% - 40\%; líneas diagonales, más de $40 \%$.

A continuación se indican los estados donde se registra algún uso no leñoso. Se presenta el número de especies utilizadas y el número total de especies reportadas para cada estado de acuerdo con González (1993), ordenados de manera creciente en función de este último valor: Tlaxcala $(2,13)$, Morelos $(9,15)$, Distrito Federal $(1,24)$, Nayarit $(2,29)$, Sonora $(1,34)$, Guerrero $(10,35)$, Michoacán $(13,38)$, Durango (1,39), Estado de México (13, 40), Chiapas $(6,44)$, Chihuahua (9, 44), Hidalgo (5, 45), Jalisco $(4,50)$, Oaxaca $(12,55)$, Puebla $(11,55)$, Veracruz $(9,57)$ y Nuevo León $(1$, 60) (figura 1). Si bien el uso se encuentra distribuido en todo el territorio nacional, la mayor proporción de especies útiles en relación al total se presenta principalmente en varios estados del centro y sur de la República, exceptuando Chihuahua. Parte de esta observación coincide con el hecho de que las montañas del centro y sur de México son las áreas más ricas en diversidad de Quercus en el hemisferio occidental (Nixon, 1998) (figura 2). Sin embargo, algunos estados del norte también presentan una gran diversidad de especies de encinos, aunque pocas son utilizadas.

Debido a esto, el número de especies utilizadas es independiente del número de especies que alberga cada entidad (figura 1). Morelos tiene el menor número de especies de encinos, pero se utiliza un alto porcentaje de ellas. Por el contrario, estados con una alta diversidad de especies como Nuevo León o Veracruz registran un número bajo de especies utilizadas. Esto significa que el uso de los encinos no está determinado por su diversidad, sino por factores de tipo cultural o por la disponibilidad de otros recursos vegetales.

Usos no leñosos. Para el análisis de los usos no leñosos se consideró lo siguiente: el uso, la parte de la planta que se aprovecha y la forma en que se utiliza.

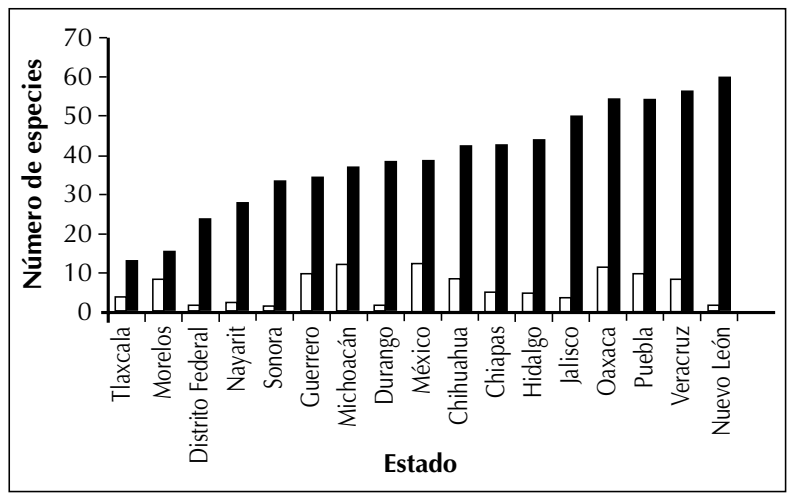

Figura 2. Relación entre las especies utilizadas reportadas por estado (barras blancas) y el número total de especies de los mismos (barras negras).

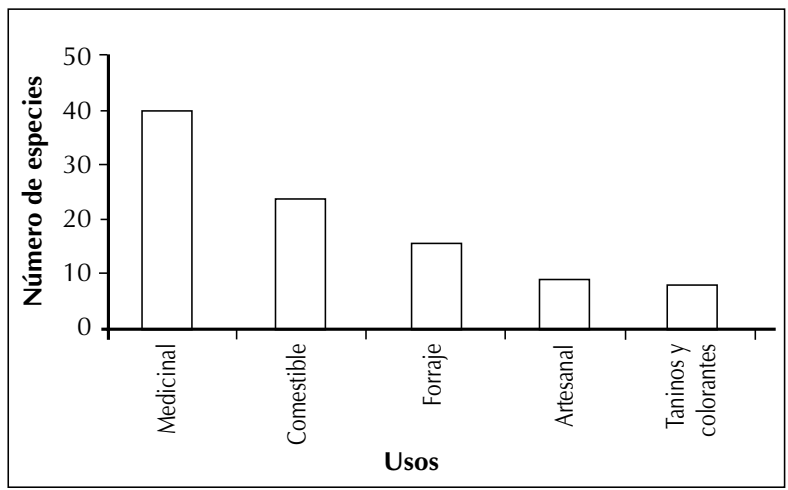

Figura 3. Número total de especies de encino utilizadas en las categorías de uso no maderable. 


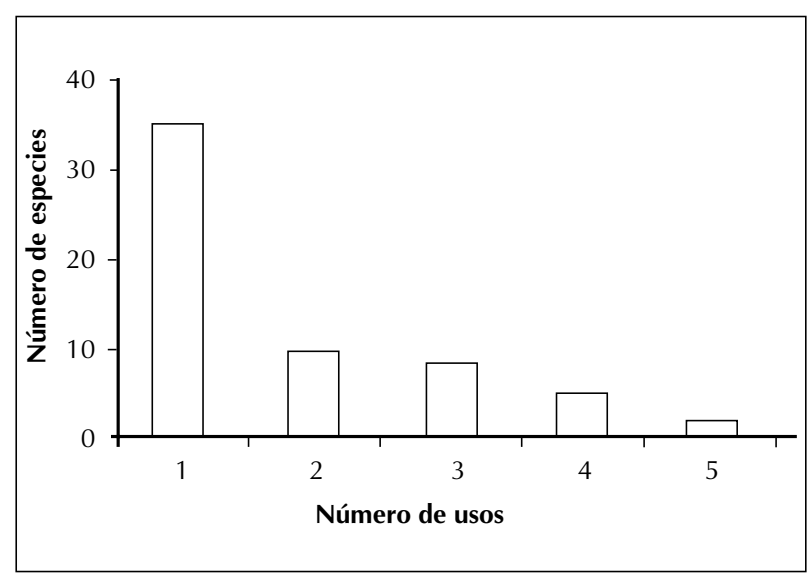

Figura 4. Frecuencia de uso de las especies de encino

Uso.- El uso se agrupó en cinco categorías: (1) medicinal, (2) alimenticio, (3) forraje, (4) artesanal, y (5) taninos y colorantes (cuadro 1). Las dos primeras categorías concentran en conjunto 53 especies, que representan $96 \%$ del total de especies utilizadas reportadas en la literatura y en los herbarios. Relativamente pocas especies se usan como forraje (25.5\%) o para elaborar artesanías (16.4\%); solamente seis especies de encino se reportan para curtir pieles $(10.9 \%)$ y una especie como mordiente o como tinte (figura 3 ).

El 64\% de las especies utilizadas de encino tienen únicamente un uso no leñoso, mientras que para las especies restantes se registraron entre dos y cinco usos (figura 4). En el segundo caso destacan algunas especies como $Q$. crassifolia,

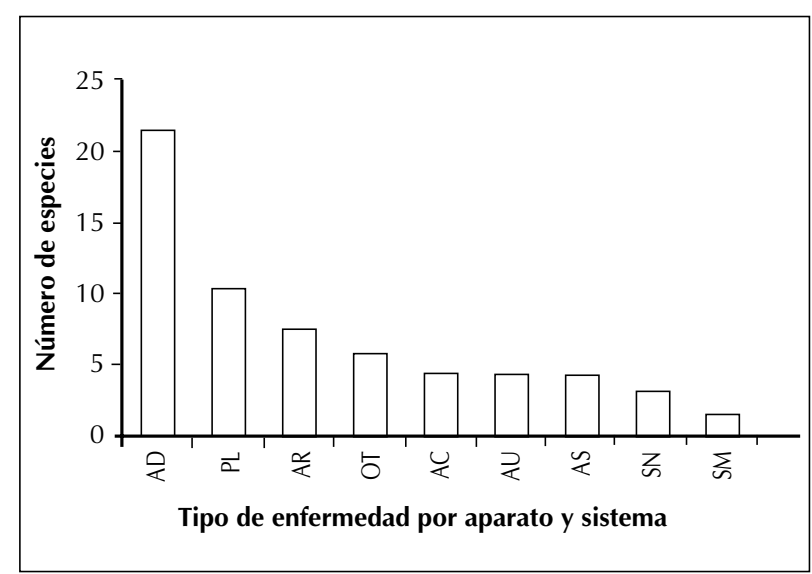

Figura 5. Número de especies utilizadas para el tratamiento de enfermedades en: $\mathrm{AD}=$ Aparato digestivo; $\mathrm{PL}=\mathrm{Piel} ; \mathrm{AR}=$ Aparato reproductor; $\mathrm{OT}=$ Otras; $\mathrm{AC}=$ Aparato circulatorio; $\mathrm{AU}=$ Aparato urinario; $\mathrm{AS}=$ Aparato respiratorio; $\mathrm{SN}=$ Sistema nervioso; $\mathrm{SM}=$ Sistema muscular.

que se utiliza como medicina y alimento, $Q$. rugosa, que es un recurso medicinal, alimenticio y de forraje, y $Q$. affinis, que se aprovecha como medicina, alimento, forraje y artesanía (cuadro 1). A continuación se describen las categorías de uso en las que se agruparon a las especies de encino, así como algunos ejemplos dentro de cada categoría.

Para el uso medicinal se emplean casi todos los órganos de la planta: corteza, hojas, flores, raíces y agallas. Se registraron 31 enfermedades relacionadas con los diferentes

Cuadro 2. Padecimientos o enfermedades tratadas con encinos.

\begin{tabular}{ll}
\hline Aparato o sistema & Padecimiento \\
\hline Aparato bucal & $\begin{array}{l}\text { Inflamación y sangrado de encías, dolor de garganta y de muelas, dientes flojos, } \\
\text { estomatitis, hematitis, úlceras bucales, gingivitis }\end{array}$ \\
Aparato digestivo & $\begin{array}{l}\text { Gastritis, dolor de estómago, diarrea, disentería, hemorragias intestinales, cáncer } \\
\text { de estómago e intestinos, inflamación intestinal, acedías }\end{array}$ \\
Piel & Heridas, granos, quemaduras, Ilagas, infección de la piel, caída del cabello \\
Aparato reproductor & $\begin{array}{l}\text { Lavados vaginales, hemorragias vaginales, desviación de la matriz, baño posparto, } \\
\text { enfriamiento después del parto, concepción, alteraciones ginecobstétricas }\end{array}$ \\
Aparato circulatorio & Corazón, circulación de la sangre \\
Aparato urinario & Mal de orín, para el dolor al orinar \\
Aparato respiratorio & Pulmones y tos \\
Sistema nervioso & Excitación nerviosa, contra los ataques \\
Sistema muscular & Dolor muscular \\
Otros & Diabetes, hemorragias
\end{tabular}


aparatos y sistemas del cuerpo humano (cuadro 2, figura 5), de las cuales las relacionadas con el aparato digestivo y la piel son las que utilizan el mayor número de especies y cuyas recetas incluyen principalmente el uso de la corteza y hojas. Algunos tratamientos se describen en el apéndice 1.

Para el uso con fines alimenticios se emplean las bellotas, las yemas foliares, las flores, las hojas y las agallas, productos que enriquecen la dieta de las comunidades indígenas con proteínas, lípidos y carbohidratos. Se encontraron diez diferentes formas de alimento proveniente de los encinos, las cuales se pueden subdividir en dos grupos. El primero comprende el consumo natural exclusivamente de la bellota; el segundo agrupa a los productos procesados tales como capeado de las flores y tostado de las bellotas para consumirse como "semilla" o para elaborar harina para tortilla, pan, pastel, atole, tesgüino, chocolate, café, pinole y tamales. Algunos ejemplos se muestran en el apéndice 1.

El forraje comprende las estructuras de las plantas de encino utilizadas para alimentar al ganado, específicamente porcino o caprino, de acuerdo con la literatura, en diferentes partes del país. Para esto, los ganaderos aportan como parte de la dieta de estos animales las bellotas y el follaje de ciertas especies de encino. El uso de bellotas de $Q$. affinis, $Q$. castanea, $Q$. candicans, $Q$. crassipes, $Q$. glabrescens, $Q$. glaucoides, $Q$. insignis, $Q$. laurina y $Q$. rugosa se reporta para los estados de Guerrero, Jalisco, Estado de México, Morelos, Oaxaca y Puebla. El uso del follaje como forraje se reporta para Nuevo León y San Luis Potosí. En esta última entidad, las hojas de encino constituyen un alimento básico para el ganado caprino durante la mayor parte del año (Zavala, 1990). En Tlaxcala, las hojas frescas de $Q$. crassipes y $Q$. obtusata son empleadas para alimentar a los burros (Espejel et al., 1999).

Para el uso artesanal, la gente de diversas comunidades aprovecha la gran variación de tamaños y formas de las bellotas de encino, además de su belleza, y produce una amplia gama de artesanías, como son los rosarios elaborados por zapotecas de Trinidad Buenavista, Oaxaca, con frutos de $Q$. candicans (Luna, 2001). Cabe mencionar otros usos artesanales, que aunque no están reportados en la bibliografía y no se tiene el nombre científico de la especie, representan productos de importancia económica para las comunidades. Tal es el caso de algunos bellotas que se utilizan en Cuetzalan (Puebla) para elaborar aretes, cuadros, cestos y juguetes; en Xochimilco (Distrito Federal) las bellotas se usan para elaborar coronas y adornos navideños con las bellotas al natural o pintadas; en Tepoztlán (Morelos) se venden collares de bellotas (I. Reyes y F. Basurto, com. pers.); en Amecameca (Estado de México) se emplean las agallas como ornamento en floreros (Estrada, 1996).

Los taninos, además de presentar propiedades medicinales, son ampliamente utilizados en el proceso de curtido de pieles y se obtienen de especies tales como $Q$. candicans, $Q$. conspersa, $Q$. crassifolia, $Q$. deserticola y $Q$. obtusata. Para fijar los colorantes únicamente se registró a $Q$. crassipes para el estado de Tlaxcala, mientras que el uso

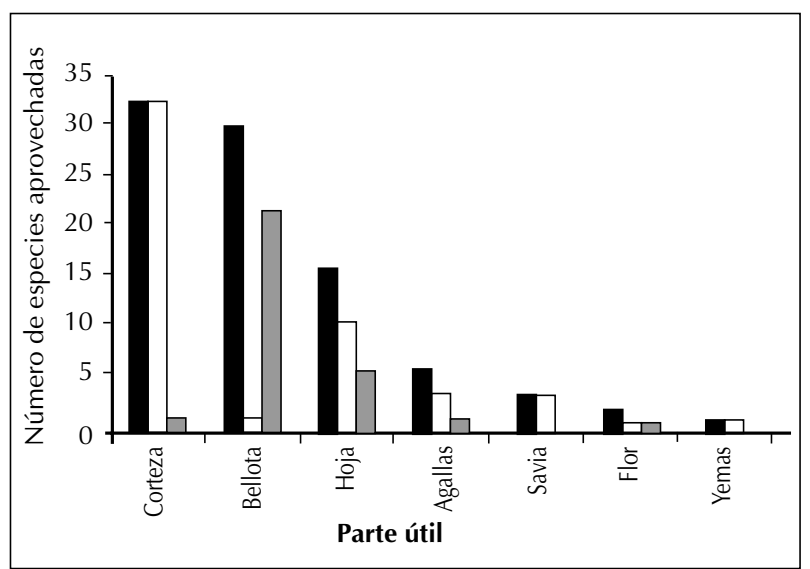

Figura 6. Relación entre la parte utilizada de los encinos y el número total de especies aprovechadas (barras negras), medicinales (barras blancas) y alimenticias (barras grises).

directo como colorante para teñir hilo de color anaranjado únicamente fue para $Q$. urbanii aff. parvifolia, registro obtenido de un ejemplar de 1952, lo que indica que este uso estaba presente hasta hace relativamente poco tiempo.

Parte utilizada de la planta. De las plantas de encino se obtiene provecho de prácticamente todas sus estructuras, tanto vegetativas como reproductivas (figura 6). Sin embargo, llama la atención que este uso integral no se da dentro de cada grupo étnico, sino que la parte útil varía entre grupos y de un estado a otro.

Las partes con mayor uso son las bellotas, la corteza y las hojas (figura 6). La elección de estas partes se debe a la mayor concentración de nutrimentos y metabolitos secundarios. Particularmente, en la corteza se concentra una gran cantidad de componentes químicos, como los taninos (ácido tanínico), que tienen propiedades de astringente, tónico y antiséptico (Ponce, 1946). Las bellotas almacenan nutrimentos importantes como carbohidratos y lípidos, por lo que se utilizan principalmente como alimento. Martínez (1959) reporta que las bellotas de $Q$. emoryi contienen $35 \%$ de aceite, $50 \%$ de almidón y 4\% de sacarosa. Vázquez (1992) indica que de esta misma especie se puede extraer un aceite con características similares al aceite de ajonjolí y cacahuate.

El análisis estadístico mostró una relación significativa entre la parte útil de la planta y el uso al que se destina $\left(\chi_{3}^{2}\right.$ g.l. $\left.=50.84, \mathrm{p}<0.05\right)$. Esto refuerza el hecho de que la mayoría de las especies se utilizan con fines medicinales, beneficio que obtienen de diferentes estructuras de la planta, mientras que el uso alimenticio se obtiene fundamentalmente de las bellotas. Cabe mencionar que de una misma especie pueden utilizarse diferentes estructuras, ya sea dentro de un mismo grupo étnico y en un mismo estado, como entre diferentes grupos étnicos (ver cuadro 1). Sin embargo, lo relevante es el papel que juega cada estructura en relación al uso. 
Grupos étnicos. Las 55 especies de encino registradas con uso no leñoso se asocian a diversos grupos étnicos del país. Las etnias que se registraron fueron, en orden creciente en términos del número de especies que utilizan: Tepehuas y Totonacos (2 especies cada uno), Mixes, Mixtecos, Purépechas y Zapotecos (4 cada uno), Otomíes (5), Mazahuas, Tarahumaras y Tepehuanes (9 especies cada uno), y Náhuatls (14).

La proporción de especies actualmente utilizadas por cada grupo étnico es muy variable y no necesariamente depende del área de distribución (a nivel estatal), ni del número total de especies utilizadas registradas por estado. El caso más obvio es el de los usos que hacen de estas especies los Tarahumaras y Tepehuanes, reportados únicamente para el estado de Chihuahua, por lo que las nueve especies que están reportadas como utilizadas necesariamente se debe a ellos. Sin embargo, en el resto de los grupos esta relación no es tan clara. Por ejemplo, los Otomíes o los Náhuatls presentan una mayor área de distribución en términos de los estados en donde se encontraron registrados y en dichos estados se registra el mayor número de especies utilizadas. Sin embargo, el porcentaje de especies utilizadas es ligeramente menor al de los Purépechas, quienes se encuentran únicamente en Michoacán, o al de los Popolucas, que se encuentran únicamente en el estado de Veracruz (figura 7). Esta información no es definitiva debido a que en particular varios de los ejemplares de herbario no necesariamente incluían información sobre este aspecto, lo que no niega la posibilidad de que existiera algún grupo étnico asociado.

Entre los grupos étnicos el uso de los encinos es variable. En Chihuahua y Guerrero la bellota forma parte de la dieta básica, aunque el grado de dependencia hacia el recurso es relativamente diferente; en Chihuahua es un recurso de uso

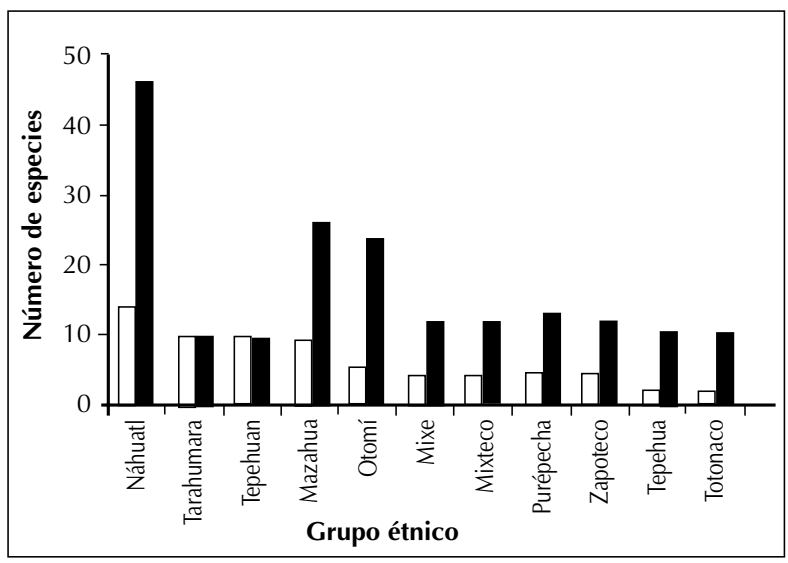

Figura 7. Relación entre el número de especies potencialmente útiles para cada grupo étnico (barras negras) y el número de especies que actualmente utilizan (barras blancas). Las especies potenciales se obtuvieron de acuerdo a la suma de especies utilizadas registradas en los estados en donde se reporta la presencia de cada grupo étnico. frecuente, mientras que en Guerrero se reporta como "alimento de emergencia" (Viveros y Casas, 1985). A pesar de ello, en ambos casos la necesidad de este recurso es tal que el $100 \%$ de las especies son utilizadas para consumo y solamente una tiene uso medicinal. En Puebla, aunque solamente se reporta el uso medicinal, las enfermedades tratadas con los encinos son de diferente índole (circulación de la sangre, dolor muscular, disentería y caída del cabello). Por el contrario, para el Estado de México fueron registradas cuatro categorías (medicinal, alimenticio, forraje y artesanal).

\section{Conclusiones}

Con base en la información obtenida de las fuentes bibliográficas y a partir de la revisión de ejemplares botánicos, se concluye que los encinos son un recurso importante para las comunidades rurales ya que, en conjunto, éstas aprovechan prácticamente cada estructura de la planta con diferentes fines. Es decir, existe un uso diversificado de este grupo de plantas, si bien en cada estado de la República y al interior de cada grupo étnico hay variaciones en el uso, las estructuras utilizadas y el número de especies aprovechadas. La diversificación de los usos con fines alimenticio, medicinal, forraje, artesanal y taninos y colorantes es reflejo del conocimiento tradicional que los diversos grupos étnicos han adquirido y conservado en relación a las especies de encino y es parte de su estrategia de subsistencia.

Debido a que el uso no leñoso de los encinos ha sido poco valorado, es importante difundir este uso, ya que además de contribuir con la sistematización de este conocimiento, se incorporan más elementos para fomentar la conservación de este recurso en nuestro país. En este sentido, sería importante analizar la composición química de las diferentes estructuras vegetales registradas en este estudio con el fin de promover el uso de estos recursos. De igual importancia resulta el análisis del contenido bromatológico de las diferentes estructuras que se utilizan como alimento. Debido a que el uso medicinal fue el más importante e incorpora varios órganos de la planta, consideramos también necesario realizar estudios fitoquímicos y farmacológicos orientados a determinar los principios activos y promover de manera formal el uso de los encinos como producto medicinal.

\section{Agradecimientos}

A la M. en C. Irma Reyes Jaramillo, la Dra. Carmen de la Paz Pérez O., la M. en C. Susana Valencia y a dos revisores anónimos, por la revisión tan minuciosa y las valiosas sugerencias al manuscrito. A la M. en C. Martha Pérez García por sugerirnos literatura relevante para el escrito. A la M. en C. Susana Valencia por la determinación de material botánico y préstamo de bibliografía, al igual que a la M. en C. Irma Reyes Jaramillo. 


\section{Literatura citada}

Adger W.N., Brown K., Cervigni R. y Moran D. 1998. Total economic value of forests in Mexico. Ambio 24:286-296.

Aguilar A., Camacho J.R., Chino S., Jácquez P. y López M.E. 1994a. Herbario Medicinal del Instituto Mexicano del Seguro Social: Información Etnobotánica. Instituto Mexicano del Seguro Social, México, D.F.

Aguilar A., Argueta A. y Cano L. 1994b. Flora Medicinal Indígena de México. Tomo I. Treinta y Cinco Monografías del Atlas de las Plantas de la Medicina Tradicional Mexicana, México, D.F.

Aguilar A., Argueta A. y Cano L. 1994c. Flora Medicinal Indígena de México. Tomo II. Treinta y Cinco Monografías del Atlas de las Plantas de la Medicina Tradicional Mexicana, México, D.F.

Aguilar A., Argueta A. y Cano L. 1994d. Flora Medicinal Indígena de México. Tomo III. Treinta y Cinco Monografías del Atlas de las Plantas de la Medicina Tradicional Mexicana, México, D.F.

Avilés F.M. 1985. Medicina tradicional: Plantas empleadas por parteras empíricas del estado de Morelos. Tesis de Licenciatura, Escuela de Ciencias Biológicas, Universidad Autónoma del Estado de Morelos, Cuernavaca, Mor., 88 pp.

Antonio B.N. 1989. Plantas medicinales utilizadas en la comunidad de San Juan Guichicoví, Oaxaca. Tesis de Licenciatura, Facultad de Biología, Universidad Veracruzana, Córdoba, 118 pp.

Bello G.M.A. y Labat J.N. 1987. Los encinos (Quercus) del Estado de Michoacán. Centre de Études Mexicaines et Centroaméricaines. Cuadernos de Estudios Michoacanos 4, Secretaría de Agricultura y Recursos Hidráulicos e Instituto Nacional de Investigaciones Forestales y Agropecuarias, México, D.F.

Berenice F.H. 2001. Aspectos ecológicos y etnobotánicos de los recursos vegetales de la comunidad Mazahua Francisco Serrato. Tesis de Licenciatura, Faculta de Biología, Universidad Michoacana San Nicolás de Hidalgo, Morelia, Mich., 137 pp.

Castro R.A.E. 1988. Estudio comparativo del conocimiento sobre las plantas medicinales utilizadas por dos grupos étnicos del municipio de Pahuatlán, Puebla. Tesis de Licenciatura, Escuela Nacional de Estudios Profesionales Iztacala, Universidad Nacional Autónoma de México, Los Reyes Iztacala, Edo. de México, 254 pp.

Chino V.S. y Jacquez R.M.P. 1986. Contribución al conocimiento de la flora medicinal de Quimixtlán, Puebla. Tesis de Licenciatura, Escuela Nacional de Estudios Profesionales Iztacala, Universidad Nacional Autónoma de México, Los Reyes Iztacala, Edo. de México, 344 pp.

de la Cruz M. y Badiano J. 1991. Libellus de Medicinalibus Indorum Herbis. Fondo de Cultura Económica e Instituto Mexicano del Seguro Social, México, D.F.

de la Paz Pérez O.C. 2000. Relación estructura propiedades fisico mecánicas de la madera de algunas especies de encinos (Quercus) mexicanos. Tesis de Doctorado, Facultad de Ciencias, Universidad Nacional Autónoma de México, México, D.F., 266 pp.

de la Paz Pérez O.C., Dávalos S.R. y Guerrero C.E. 2000. Aprovechamiento de la madera de encino en México. Maderas y Bosques 6:3-13.

Espejel-Rodríguez M.M.A., Santacruz-García N. y Sánchez-Flores M. 1999. El uso de los encinos en la región de La Malinche, Estado de Tlaxcala, México. Boletín de la Sociedad Botánica de México 64:35-39.
Estrada M.E. 1996. Etnobotánica forestal en Santa Isabel Chalma, Amecameca, México. Tesis de Maestría en Ciencias, Programa de Botánica, Colegio de Posgraduados, Montecillo, Edo. de México, 270 pp.

Food and Agriculture Organization (FAO). 1999. Hacia una definición uniforme de los productos forestales no madereros. Unasylva 50:63-66.

García H.M.C. 1981. Plantas medicinales utilizadas para la diabetes en los mercados de Monterrey, N.L. México. Tesis de Licenciatura, Facultad de Ciencias Biológicas, Universidad Autónoma de Nuevo León, Monterrey, N.L., 140 pp.

García G.B.I. 1992. Estudio etnobotánico de las cortezas medicinales del estado de Morelos y zonas limítrofes con Puebla y Guerrero. Tesis de Licenciatura, Universidad Autónoma del Estado de Morelos, Cuernavaca, Mor., 114 pp.

Gómez M.S. 2000. Estudio etnobotánico de la flora útil del municipio de Nuevo Urecho, Michoacán. Tesis de Licenciatura, Escuela Nacional de Estudios Profesionales Iztacala, Universidad Nacional Autónoma de México, Los Reyes Iztacala, Edo. de México, 117 pp.

Gómez S.L.C. y Chong C.I. 1985. Conocimiento y usos de la flora de Amatlán, municipio de Tepoztlán, Morelos. Tesis de Licenciatura, Facultad de Ciencias, Universidad Nacional Autónoma de México, México, D.F., 185 pp.

González V.L.M. 1986. Contribución al Conocimiento del Género Quercus (Fagaceae) en el Estado de Jalisco. Instituto de Biología, Universidad de Guadalajara, Guadalajara.

González R.R. 1993. La diversidad de los encinos mexicanos. Revista de la Sociedad Mexicana de Historia Natural Vol. Esp. (XLIV):125-142.

Govaerts F. y Frodin D.G. 1998. World Checklist and Bibliography of Fagales (Betulaceae, Corylaceae, Fagaceae and Ticodendraceae). Royal Botanic Gardens, Kew.

Jiménez A.C.D. 1990. Catálogo de plantas comestibles en la República Mexicana. I. Árboles, arbustos y otras leñosas. Informe del Servicio Social, Universidad Autónoma Metropolitana - Xochimilco, México, D.F.

López V.M.E. 1988. Contribución etnobotánica en plantas medicinales utilizadas por dos grupos étnicos de Mecapalapa, municipio de Pantepec, Puebla. Tesis de Licenciatura, Escuela Nacional de Estudios Profesionales Iztacala, Universidad Nacional Autónoma de México, Los Reyes Iztacala, Edo. de México, 349 pp.

Luna B.J.F. 1989. Estudio de las plantas utilizadas en el baño de temascal en la medicina tradicional de Morelos. Tesis de Licenciatura, Facultad de Ciencias, Universidad Nacional Autónoma de México, México, D.F., 327 pp.

Luna J.A.L. 2001. Análisis del conocimiento etnobotánico entre los zapotecos de la comunidad Trinidad Buenavista Loxicha, Oaxaca. Informe del Servicio Social, Universidad Autónoma Metropolitana-I., México, D.F.

Maldonado A.B.J. 1997. Aprovechamiento de los recursos florísticos de la Sierra de Huautla, Morelos, México. Tesis de Maestría en Ciencias, Facultad de Ciencias, Universidad Nacional Autónoma de México, México, D.F., 149 pp.

Martínez M. 1959. Plantas Útiles de la Flora Mexicana. Ediciones Botas, México, D.F.

Martínez M.M.E. 1992. Especies vegetales como recurso energético de uso doméstico, en Zozocolco de Hidalgo, Veracruz. Tesis de Licenciatura, Facultad de Ciencias, Universidad Nacional 
Autónoma de México, México, D.F., 122 pp.

Martínez M. 1994. Catálogo de Nombres Vulgares y Científicos de Plantas Mexicanas. Fondo de Cultura Económica, México, D.F.

Morales G.G. y Toledo O.G. 1987. Contribución al estudio de la flora medicinal y medicina tradicional del municipio de Coxquihui, Veracruz. Tesis de Licenciatura, Facultad de Ciencias, Universidad Nacional Autónoma de México, México, D.F., 307 pp.

Negrete F.F. 1991. Etnobotánica de las plantas empleadas en afecciones de la piel en Xochipala, Guerrero. Tesis de Licenciatura, Facultad de Ciencias, Universidad Nacional Autónoma de México, México, D.F., 94 pp.

Nixon K.C. 1998. El género Quercus en México. En: Ramamoorthy T.P., Bye R., Lot A. y Fa J. Eds. Biodiversidad Biológica de México: Orígenes y Distribución, pp. 435-448, Instituto de Biología, Universidad Nacional Autónoma de México, México, D.F.

Ponce. 1946. Los Encinos en el Circuito Montañoso del Valle de México. Instituto de Biología, México. D.F.

Reyes J.I. 1995. Los bosques de encino en México: riqueza que se convierte en carbón. Contactos 8:28-35.

Salgado M.S. 1978. Etnobotánica mexicana: plantas popularmente usadas en el Estado de México para el tratamiento del aparato digestivo. Tesis de Licenciatura, Facultad de Ciencias, Universidad Nacional Autónoma de México, México, D.F., $81 \mathrm{pp}$.

Sandoval A.M. 1977. Etnobotánica mexicana: las plantas medicinales utilizadas en Tulancingo, Hidalgo. Tesis de
Licenciatura, Facultad de Ciencias, Universidad Nacional Autónoma de México, México, D.F., 107 pp.

Sánchez T.V. 1996. Plantas medicinales del municipio de Ixtenco, Tlaxcala, México. Tesis de Licenciatura, Departamento de Agrobiología, Universidad Autónoma de Tlaxcala, Tlaxcala, $101 \mathrm{pp}$.

Valencia A.S. 1995. Contribución al Conocimiento del Genero Quercus (Fagaceae) en el Estado de Guerrero, México. Contribuciones del Herbario de la Facultad de Ciencias, No. 1, Universidad Nacional Autónoma de México, México, D.F.

Vázquez V.M.L. 1992. El género Quercus (Fagaceae) en el estado de Puebla. Tesis de Licenciatura, Facultad de Estudios Superiores Zaragoza, Universidad Nacional Autónoma de México, México, D.F., 246 pp.

Viveros S.J.L. y Casas F.A. 1985. Etnobotánica mixteca: alimentación y subsistencia en la montaña de Guerrero. Tesis de Licenciatura, Facultad de Ciencias, Universidad Nacional Autónoma de México, México, D.F., 141 pp.

Zamora M.M.C. y Hernández L. 1985. Catálogo de especies de plantas útiles con importancia económica de la región norte de los estados de Puebla y Veracruz. Ciencia Forestal 10:16-32.

Zavala C.F. 1990. Los encinos mexicanos: un recurso desaprovechado. Ciencia y Desarrollo 18:43-51.

Zavala C.F. 1998. Observaciones sobre la distribución de encinos en México. Polibotánica 8:47-64.

Zavala C.F., Estrada E.E. y Arriola P.V. 1999. Los Encinos del Herbario de la Universidad Autónoma de Chapingo. Universidad Autónoma de Chapingo, Chapingo, Edo. de México.

Fecha de recepción: 30 de agosto de 2002

Versión corregida: 12 de mayo de 2003

Aceptado: 30 de mayo de 2003 
Apéndice 1. Ejemplos de algunas especies de encino que se usan como medicina o alimento en México. Se describe brevemente la forma de procesamiento de las estructuras utilizadas de las plantas.

\section{Medicinal \\ Comestible}

Quercus acatenangensis. En Motozintla (Chiapas) se usa la corteza y las hojas para curar heridas, llagas y granos infectados. Se hierven en agua y posteriormente al enfriarse ésta, se lavan y desinfectan las lesiones (Aguilar et al., 1994d). El mismo procedimiento se sigue en casos de infecciones vaginales, para lo cual también se cita el uso de $Q$. oleoides.

Quercus acutifolia. En Xochipala (Guerrero) los mestizos secan la corteza, la muelen y el polvo lo aplican en la piel en caso de quemaduras (Negrete, 1991).

Quercus conspersa. En Quimixtlán (Puebla) los náhuatls utilizan la corteza para apretar dientes y desinflamar encías (Aguilar et al., 1994b).

Quercus crassifolia. En Morelos su corteza se utiliza junto con otras plantas en el temazcal para bañar a las mujeres después del parto (Luna, 1989). En Chihuahua los tarahumaras emplean las hojas de esta misma especie, aplicándolas calientes como tratamiento para la inflamación de las amígdalas (Aguilar et al., 1994b).

Quercus aff. obtusata. En el Estado de México se utilizan las hojas para tratar la diabetes.

Quercus resinosa. En Nuevo Urecho (Michoacán) los purépechas emplean las agallas conocidas como "borreguitos" para el tratamiento de la tos. Se hierven en agua y su aplicación es oral (Gómez, 2000).

Quercus magnoliifolia. En Nayarit se utilizan las agallas como parte de las prácticas terapéuticas en el tratamiento de heridas de personas y animales. Se tuestan, muelen y aplican en la región afectada (Aguilar et al., 1994c).
Quercus emoryi por los tarahumaras en Chihuahua (Linares, 1994); Q. glaucoides y Q. obtusata por los mixtecos en Alcozauca, Guerrero (Viveros y Casas, 1985); Q. albocincta, Q. arizonica, Q. chihuahuensis, $Q$. durifolia, $Q$. hypoleucoides y $Q$. sideroxyla por los tepehuanes en Chihuahua (Jiménez, 1990) consumen las bellotas al natural o tostadas.

El consumo de las bellotas y otras estructuras procesadas se ejemplifica con Quercus rugosa. En Chihuahua los tepehuanes tuestan las bellotas para elaborar tamales, atole, café y chocolate. En Jalisco también se elabora café a partir de las bellotas. Las yemas de las hojas se muelen y mezclan con la masa de maíz para hacer tortillas (Jiménez, 1990).

Quercus crassipes. En el Estado de México los otomíes consumen las flores hervidas y capeadas con huevo (Jiménez, 1990).

Quercus crassifolia. En Chihuahua los tepehuanes utilizan las hojas y corteza para elaborar la bebida conocida como tesgüino (Jiménez, 1990).

Quercus laurina y Quercus obtusata. En el Estado de México las agallas se consumen verdes (Linares, No.col. 738). 